\title{
Pratiques ethno-vétérinaires sur les éléphants au
}

\section{Laos}

Un savoir co-construit avec les animaux?

ethnoveterinary practices on elephants in Laos: a knowledge co-constructed with animals?

\section{Nicolas Lainé}

\section{OpenEdition}

\section{Journals}

\section{Édition électronique}

URL : http://journals.openedition.org/ethnoecologie/5917

DOI : $10.4000 /$ ethnoecologie. 5917

ISSN : 2267-2419

\section{Éditeur}

Laboratoire Eco-anthropologie et Ethnobiologie

\section{Référence électronique}

Nicolas Lainé, «Pratiques ethno-vétérinaires sur les éléphants au Laos », Revue d'ethnoécologie [En ligne], 17 | 2020, mis en ligne le 30 juin 2020, consulté le 03 juillet 2020. URL : http:// journals.openedition.org/ethnoecologie/5917 ; DOI : https://doi.org/10.4000/ethnoecologie.5917

Ce document a été généré automatiquement le 3 juillet 2020

\section{(c) (i) (9)}

Revue d'ethnoécologie est mis à disposition selon les termes de la licence Creative Commons Attribution - Pas d'Utilisation Commerciale - Pas de Modification 4.0 International. 


\title{
Pratiques ethno-vétérinaires sur les éléphants au Laos
}

\author{
Un savoir co-construit avec les animaux? \\ ethnoveterinary practices on elephants in Laos: a knowledge co-constructed with \\ animals?
}

Nicolas Lainé

\section{Introduction}

Cet article fait état des connaissances médicinales des cornacs et des spécialistes de la santé des éléphants en contexte rural laotien. Il se base sur une enquête ethnographique ${ }^{1}$ conduite dans les villages Tai-Lue et Tai-Lao dans la province de Sayabouri au Nord-Ouest du pays. Un travail préliminaire portant sur la perception locale des maladies affectant les pachydermes (Lainé 2017a) a souligné d'importants changements quant aux à la gestion et aux soins apportés à ces animaux dans le pays. Sous l'égide d'une ONG étrangère, implantée à l'aune des années 2000, la médicalisation occidentale (allopathique) s'est rapidement imposée dans les parcs touristiques et les centres de conservation de l'espèce (Labatut \& Suter, 2010). Ces institutions, récentes dans l'histoire des relations homme-éléphant, emploient aujourd'hui majoritairement les éléphants du pays, en les louant de manière saisonnière ou en les achetant à leurs propriétaires. Au niveau villageois pourtant, les changements induits ne font pas l'unanimité parmi les premiers intéressés, cornacs et propriétaires. Pour preuve, tandis que depuis le début des années 2000, sous l'impulsion de l'ONG occidentale, une unité mobile vétérinaire est disponible gratuitement pour venir visiter les éléphants souffrants, peu de cornacs et propriétaires d'animaux me confiaient faire appel à eux, préférant consulter les spécialistes au village ou tout simplement laisser leurs éléphants se soigner eux-mêmes. Pour certains mêmes, les camps de touristes ou centres sont considérés comme une prison pour les éléphants, car selon leurs cornacs, les animaux n'y ont pas la possibilité de vaguer librement pour aller rechercher des 
essences spécifiques pour se soigner eux-mêmes comme c'est le cas lorsqu'ils vivent au village.

Comme nous le verrons, au village, cet aspect de la gestion de la santé est considéré comme partie intégrante du système de soin apporté à ces animaux. Aux dires de ces derniers les éléphants seraient capables de se soigner seuls par automédication ${ }^{2}$. Ces informations, jamais contredites durant l'enquête, m'ont alors invité à élargir le corpus de données pour relever des informations sur le régime alimentaire des pachydermes, en lien notamment avec leur santé.

D'un point de vue méthodologique, le travail de terrain a mobilisé les outils propres à l'enquête ethnographique qui implique immersion auprès de la population concernée, observation répétée de pratiques et leurs variantes, ou encore l'observation, et la conduite d'entretiens semi directifs et de récits de vie. Sur le terrain, j'ai également mobilisé les outils et méthodes des ethnosciences, notamment l'ethnobotanique et l'ethnozoologie (Hunn 2012). Au total, 36 cornacs et propriétaires d'éléphants ont été interrogés dans le Nord de la province de Sayabouri, principalement dans les villages avoisinants la ville de Hongsa (Figure 1). Dans cette province, vivent encore aujourd'hui un certain nombre d'éléphants de village, mais aussi de forêt.

Figure 1 : Situation du terrain d'enquête et répartition des éléphants au Nord-Ouest du Laos

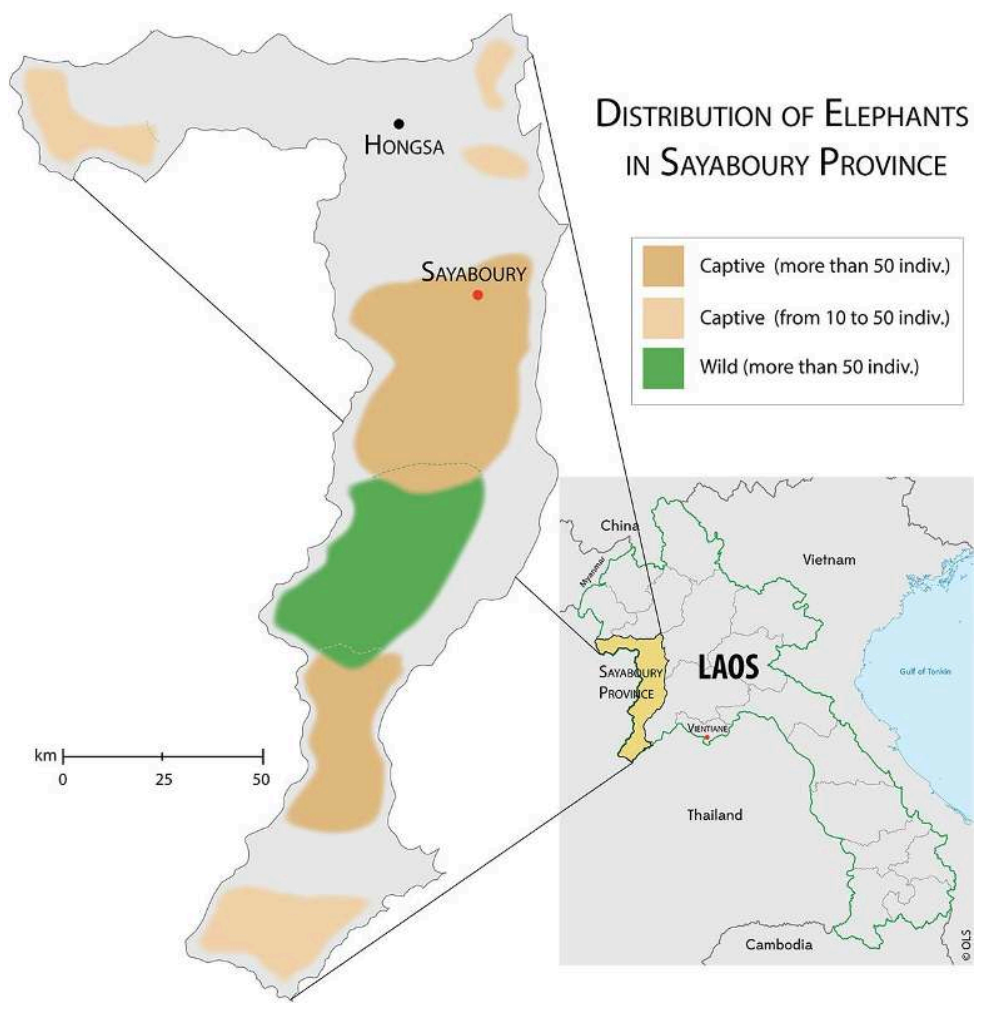

L'exploration du régime alimentaire des pachydermes s'est inspirée de la démarche ethno-éthologique telle que développée par Florence Brunois (2005). Pratiquer l'ethnoéthologie, écrit-elle, incite à conduire une « ethnographie de la façon dont les individus perçoivent et conçoivent, dans le cours de leur interaction avec eux, le comportement des êtres vivants et la manière dont ils réagissent à ces comportements " (Brunois 2005 : 34). Sur le terrain, cela signifie accéder aux savoirs et aux connaissances des 
pachydermes sur leur environnement par la médiation des cornacs, en particulier sur la manière dont ils perçoivent les dits-comportements. J'ai donc d'abord interrogé les cornacs sur leurs connaissances des plantes consommées par les animaux, j'ai également effectué des sorties en forêt en leur compagnie. Il s'agissait d'observer directement les plantes ou toute autre ressource végétale (racine, branche, fruit, feuille, liane, écorce) consommées par les pachydermes. Ce pouvait être le matin, en allant chercher l'animal, qui est généralement laissé libre le soir dans la forêt environnante, ou durant leur activité quotidienne au travail.

Après avoir exposé un ensemble de données ethnographiques sur les pratiques locales en termes de santé pour les éléphants, je présenterai les informations collectées sur l'alimentation des éléphants, toujours en lien avec leur santé. Cette seconde partie permettra d'initier la discussion et d'émettre des hypothèses sur le partage et la coconstruction de savoirs entre humains et éléphants en conclusion.

\section{Savoirs sur les pachydermes}

6 Au Laos, les éléphants de village (xang ban) bénéficient d'un système de gestion et de soins impliquant d'abord les cornacs et les propriétaires des animaux. En matière de santé, ces derniers, qui les côtoient et les connaissent très bien, sont attentifs aux différents signes émis par ceux-ci. Pour les soins quotidiens, ils disposent d'un ensemble préparations transmises de manière informelle de génération en génération par les membres de leurs familles. Et, selon la nature du problème rencontré par les animaux, ils font appel à des spécialistes : les mo. Au Laos, ce terme mo renvoie à toute personne douée d'un talent, d'un savoir ou d'un pouvoir déterminé. Il regroupe les médecins, magiciens, astrologues ou devins (Pottier 1973). Certains de ces spécialistes soignent par incantations les mo phi, d'autres par l'usage de plantes, les mo ya.

7 Dans cet aperçu des soins prodigués aux éléphants au village, je propose de reprendre la distinction opérée par Pottier (2007) entre médecine des rituels (les mo phi) et médecine des remèdes (les mo $y a$ ), que je complèterai par les connaissances directement mises en œuvres par les cornacs.

\section{Médecine des rituels}

8 Les mo phi ont recours de manière exclusive à des traitements rituels, principalement par incantations. Notons que dans le cas spécifique du traitement des éléphants, on parlera de mo xang («spécialiste des éléphants »). De manière générale, les mo xang se basent sur une interprétation de la maladie en termes d'agression de mauvais esprits, de sanction ou d'infortune. Aussi, avant de devenir mo, chacun a été initié par des enseignements soit à la pagode durant leur enfance, soit par un membre de leur famille. Au terme de cette initiation, ils établissent une relation contractuelle avec les esprits (phi) qui sont omniprésents dans le quotidien des Tai-Lue ou des Tai-Lao.

$9 \mathrm{Au}$ village, chaque éléphant est considéré comme appartenant au foyer de son propriétaire, dont il est membre à part entière au même titre que l'ensemble des membres. Ainsi, chaque éléphant de village (xang ban) vit sous la protection de l'esprit $\mathrm{du}$ foyer de son habitation d'appartenance, le phi huean. Et chaque fois qu'un propriétaire quitte son habitation pendant plusieurs jours, notamment pour aller travailler avec l'animal dans la forêt, il en informe son phi huean, et lui demande par 
une prière protection pour lui et son éléphant. En plus de son rôle protecteur, le phi huean a la capacité d'agir directement sur la santé ou le comportement des pachydermes, en fonction des relations sociales entre les hommes. Il n'est pas rare au Laos que les éléphants aient plusieurs propriétaires. À la mort des parents, les animaux sont transmis à l'ensemble des enfants qui en deviennent les propriétaires partagés. Or, l'esprit de chacun des parents reste attaché au phi huean du foyer, allant jusqu'à plusieurs générations. Aussi, il arrive que le phi huean d'un foyer soit irrité par la façon dont est géré et maintenu l'animal, ou même tout simplement mécontent des relations entretenues par les enfants de la famille. Durant mes enquêtes, j'ai collecté plusieurs informations au sujet de querelles, disputes ou jalousies à l'intérieur d'un même foyer et de leurs conséquences sur le comportement de l'éléphant. Il arrive par exemple que l'animal n'écoute pas ou ne veut pas travailler, ou même qu'il disparaisse pendant plusieurs jours dans la forêt sans que personne ne puisse le retrouver. Dans ces cas, la raison première évoquée renvoie au phi huean, lequel aurait volontairement caché l'animal privant ainsi la famille de revenus. Afin de faire revenir l'animal, ou bien pour que celui-ci retrouve un comportement attendu, les enfants doivent alors faire un vœu au phi huean par une cérémonie (van phi) et lui promettre de ne plus se disputer. Cela peut inclure une offrande sacrificielle, lorsque l'on a retrouvé l'animal.

On le voit, le phi huean a un rôle important pour le quotidien des relations entre les hommes et les éléphants. Si l'animal est vendu, il faut d'abord qu'il soit séparé de son phi huean d'origine et accepté par celui de son futur propriétaire. Si cette opération, qui passe par une cérémonie rituelle, n'est pas réalisée l'animal cherchera toujours à revenir dans son ancien foyer et n'écoutera pas son nouveau cornac. De même, si l'éléphant est vendu dans un autre village, son nouveau propriétaire doit également effectuer une même cérémonie pour s'adresser à l'esprit protecteur du village (le phi ban) afin de l'avertir de la présence d'un nouvel être, avant d'informer son phi huean qu'il y a désormais un éléphant au sein du foyer, dont il faut s'occuper et protéger ${ }^{3}$.

De même selon la cosmologie des Tai-lue et Tai-lao, outre les esprits, les éléphants, comme les hommes, sont animés par une force vitale, les khwan. Ces croyances impliquent une forte ritualisation du quotidien des relations entre les hommes et les éléphants. Celle-ci s'exprime, par exemple, chaque soir lorsque les cornacs laissent leur éléphant dans la forêt après une journée de travail. À ce moment, ils informent l'esprit de la forêt (phi pa) ainsi que le dieu du sol et de la terre du territoire spécifique (chao don chao dee) de la présence de l'animal sur leur territoire, et leur demandent d'en prendre soin, en le protégeant en cas d'attaque par d'autres animaux - comme les serpents -, mais aussi par des mauvais esprits (phi phai).

12 À l'époque où la capture des éléphants se pratiquait encore ${ }^{4}$, ces spécialistes étaient indispensables pour le bon déroulement des opérations. Selon la méthode du piège à fosse $^{5}$ (khoum xang) employée dans le Nord-Ouest du pays, les mo xang avaient la responsabilité de ramener l'animal capturé au village, de veiller à ce qu'il ne soit pas poursuivi ni par la mère de l'animal, ni par des esprits malveillants. Avant de faire sortir l'animal de la fosse dans laquelle il a été pris, ce spécialiste doit d'abord s'assurer que des mauvais esprits ne sont pas présents dans le corps de l'éléphant. Il doit également empêcher de nouveaux esprits de venir attaquer les animaux. Une fois sorti du khoum xang, ce spécialiste s'engage dans un combat symbolique avec le phi kuhan xang pa, le cornac symbolique de l'éléphant de forêt qu'il doit faire fuir, afin que l'animal puisse avoir un futur cornac humain (Lainé 2018c). 
13 Les mo xang sont ensuite appelés au moment du dressage ${ }^{6}$ des éléphants. Là encore leur présence permet de s'assurer que l'éléphant passe cette épreuve et accepte ses nouvelles conditions d'existence. Au Laos, cette opération qui mobilise l'ensemble du village commence par une cérémonie présidée par le mo xang qui consiste à éloigner les esprits sauvages qui pourraient encore habiter l'animal, mais aussi à apaiser les esprits du village. S'il s'agit d'une naissance au village, il doit au préalable séparer la mère du jeune éléphanteau ${ }^{7}$. Lung $\mathrm{Si}^{8} \mathrm{~m}^{\prime}$ indique qu'avant d'entreprendre cette opération, le spécialiste lance des œufs' ${ }^{9}$ au-dessus de la mère. Si l'œuf ne se casse pas en retombant au sol, il ne peut commencer le dressage. L'opération se déroule dans une petite hutte construite pour l'occasion, le khok xang, dans laquelle sera logé l'animal. Le mo xang purifie à l'aide d'eau bénite (sum poi) les équipements nécessaires (notamment des cordes en peau de buffle). Parmi les différentes opérations ${ }^{10}$, un poulet est relâché sur le dos de l'animal et emportera dans sa fuite les mauvais esprits avec lui. Finalement, lorsque l'animal accepte la présence humaine et notamment d'être monté sur son cou (au bout de trois ou quatre semaines), le mo xang fera choisir son nom à l'éléphant en attrapant avec sa trompe un morceau de canne à sucre parmi plusieurs où sont gravés différents noms choisis par son propriétaire (Maurer 2018).

Ensuite, tout au long de la vie de l'animal, en cas d'attaque d'un mauvais esprit, les cornacs ou propriétaires consultent le mo xang qui agit comme un exorciste. Par des incantations (khatha), ce spécialiste rituel va rentrer en contact avec le phi phai qu'il va chasser du corps de l'éléphant. De même, chaque année, les propriétaires font également appel aux mo xang pour réaliser le baci (ou sukhwan) aux éléphants, au moment du nouvel an lao généralement en avril. Cette cérémonie, qui rassemble l'ensemble des propriétaires de l'éléphant mais aussi les membres de la famille se déroule en trois étapes. Elle se tient habituellement dans l'enceinte du foyer, et nécessite un ensemble d'offrandes, composée de riz, de poulet mais aussi de l'alcool ou des cigarettes. Au préalable, la cérémonie commence par une purification de l'éléphant l'aide d'eau bénite. Il s'agit d'abord de chasser les mauvais esprits du corps des éléphants, puis de rappeler et rassembler les khwan qui seront attachés avec du fil blanc, sur les pattes, oreilles et la trompe de l'animal (Figure 2). Chaque membre du foyer est invité à aller attacher un morceau de fil sur l'éléphant. Ces fils sont ensuite reliés et tenus par le mo xang qui va prévenir les khwan et les nourrir. Il opère en soufflant sur les fils et en murmurant dessus. Il s'agit enfin de faire des vœux et de souhaiter une bonne santé à l'animal. À la fin l'ensemble du buffet est partagé par les hommes tandis que les animaux se voient offrir des morceaux de canne à sucre. Cette même cérémonie peut être réalisée durant l'année pour remercier l'animal pour le travail et les services rendus aux hommes.

Rajoutons que la cérémonie du baci est aujourd'hui encore très courante au Laos. Elle concerne également les hommes (Zago 1972), mais aussi d'autres animaux comme les buffles. Ce dernier point et les autres présentés en amont soulignent qu'il y a au Laos une correspondance entre le traitement rituel des humains et celui des éléphants. Par ailleurs, les éléments présentés ci-dessus montrent une forte ritualisation du quotidien des relations entre les hommes et les éléphants au village. Pour veiller à maintenir l'équilibre de ces relations, le mo xang a un rôle prépondérant, depuis la capture et l'intégration d'un animal au village et dans son foyer jusqu'à la mort de l'animal, où il sera à nouveau chargé de séparer l'éléphant de son foyer et d'empêcher son esprit de venir semer le trouble au sein de celui-ci. 
Figure 2 : Lors d'un baci, le mo xang entoure les pattes d'un éléphant avec du fil blanc

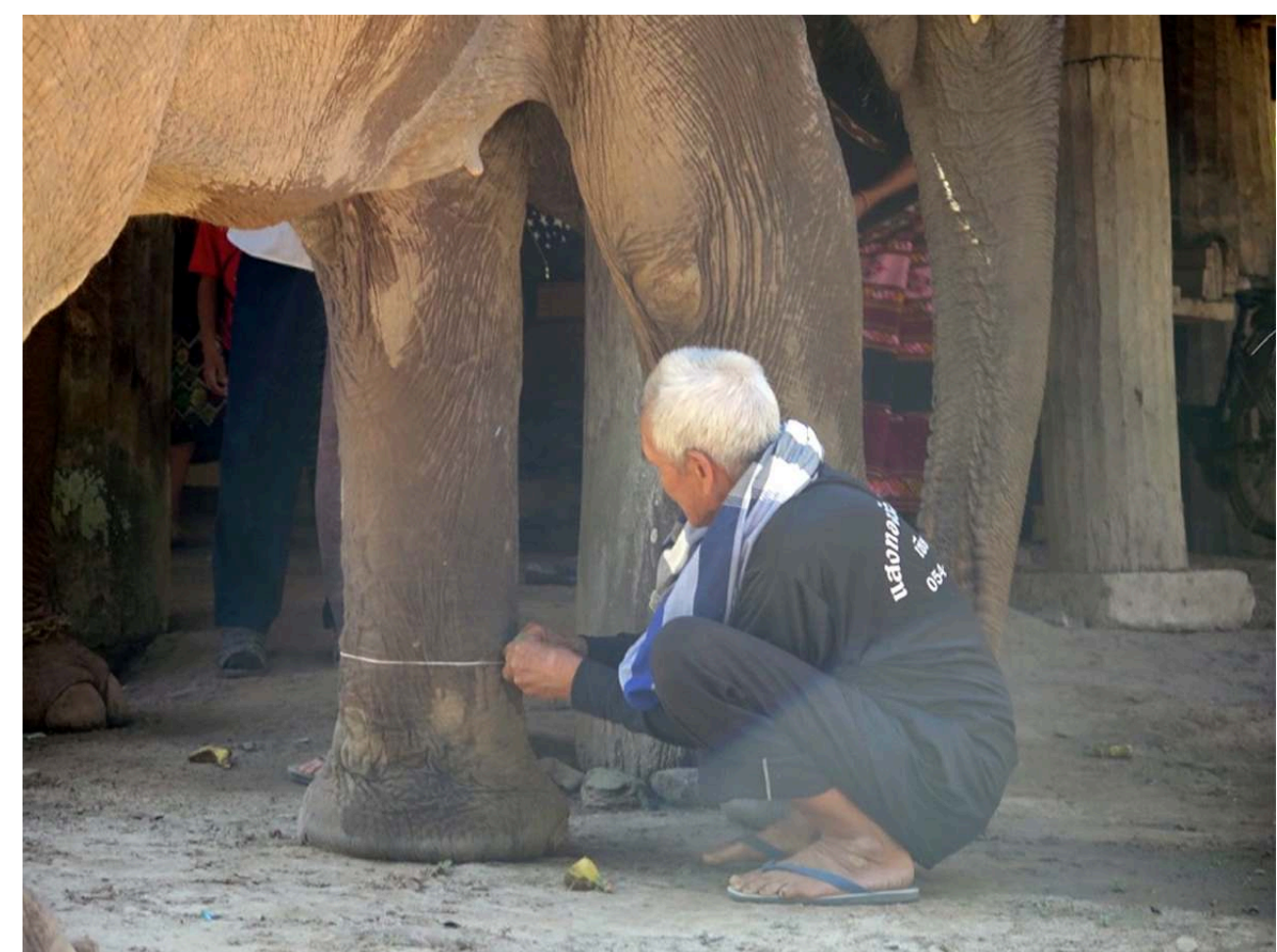

(c) N. Lainé 2016

\section{Médecine des remèdes}

$16 \mathrm{Au}$ village, il existe d'autres guérisseurs pour veiller au bien-être et à la santé de pachydermes, les mo ya. Ces derniers soignent les hommes et les animaux à l'aide de remèdes composés en grande majorité par des plantes. Ainsi que le rappelle Jules Vidal à propos des pratiques thérapeutiques laotiennes, « on peut affirmer que les 9/10e des substances employées dans l'art de guérir sont d'origine végétale» (Vidal 1958:602). Richard Pottier (2007) précise quant à lui que ces spécialistes s'appuient sur une interprétation de la maladie en termes de désordre organique, des quatre éléments (terre/eau/ souffle/ canaux où l'ensemble circule).

17 L'ensemble des recettes utilisées par les mo ya est répertorié dans des codex thérapeutiques, nommés tamla ya, littéralement « traité sur les plantes ». Dans le village de Viengkeo, j'ai rencontré Noi Lue qui dispose de plusieurs tamla, dont il a directement hérité de son père (un spécialiste réputé dans la région, mais dont il n'a pas souhaité hérité de son pouvoir). Parmi les nombreux manuscrits (sept au total) dont il dispose, certains concernent précisément la cérémonie du baci (cf. infra), ou encore le dressage des animaux. Un autre non considéré comme tamla mais plutôt comme un manuel (phum) où sont regroupés et classés différents types d'éléphants, selon des attributs physiologiques associés à un ou plusieurs traits de caractère propre pour chaque animal, le phum gnok xang. Noi Lue possède par ailleurs un traité dédié aux plantes servant à soigner les éléphants. Sur la couverture, il est indiqué que les recettes mentionnées dans ce manuscrit peuvent s'appliquer à d'autres animaux comme les chevaux ou les buffles. Ce traité de Noi Lue décrit un ensemble de compositions pour les 
soins quotidiens avec, généralement, une ou plusieurs variantes si la première n'agit pas correctement. On trouve par exemple des compositions pour lutter contre la constipation, d'autres si les éléphants ont les pattes endolories, lorsqu'ils ont la mâchoire bloquée, lorsqu'ils ont des irritations sur la peau, ont perdu l'appétit, mal à la gorge, ou encore lorsque les animaux montrent des signes de faiblesse ou une tension basse. Les lignes à suivre présentent quelques compositions extraites de ce tamla.

Pour traiter les abcès formés par le frottement des différents cordes nécessaires au travail en forêt, il est indiqué de faire d'abord bouillir des racines de nam hanh (Acacia concinna), puis de laver la peau de l'éléphant avec. Il s'agit ensuite de frotter l'abcès avec de l'écorce de manguier, mak kok (Spondias pinnata), et de laisser sécher. Cette opération doit être renouvelée quotidiennement jusqu'à ce que l'abcès dégonfle.

Sur la même page est indiquée une préparation qui aiderait les éléphants à retrouver l'appétit s'ils sont tristes et ne souhaitent pas manger. Pour cela, la composition indique de mélanger sept feuilles de gingembre (khing), sept feuilles de piment (mak phet khee), deux cigales, huit pattes d'une araignée (southeast asia black tarantula, de la famille des Theraphosidae), et du tamarin (mak kham). L'ensemble doit être d'abord pilé, puis formé en boules qui seront données à manger à l'animal le matin au moment d'aller le chercher dans la forêt.

Une autre préparation est indiquée pour faire baisser la tension des éléphants. Pour cela, le traité indique de piler ensemble du galanga (hua kha) et de l'écorce de tamarin (puek mak kham), puis de rajouter du sel et de la canne à sucre noire (oi dam). De la même manière que pour la préparation précédente l'ensemble, une fois formé en pate épaisse, doit être constitué en boules qui seront directement données à l'animal.

Est également mentionnée une préparation, composée de dix ingrédients, qui favoriserait la montée de lait des femelles. Il s'agit d'une véritable préparation vitaminée qui inclut des figues (mak deua), fruits de la passion (mak nod), bananes (kwai); de pomme-éléphant (Dillenia obovata); des feuilles d'épinard (Lasia spinosa); du tamarin ; de la canne à sucre noire (oi dam); du riz blanc cuit (khao chao); du miel (nam pheung) et du lait maternel humain (nam nom khon). L'ensemble doit être coupé en petit morceaux, puis séché avant de constituer des boules qui seront données à la femelle allaitante.

22 Le traité de Noi Lue indique également une préparation vitaminée pour les pachydermes, nommée ya bam loung (littéralement "boule de vitamines de plantes »), pour donner de l'énergie aux éléphants. Cette préparation se compose d'une quinzaine d'ingrédients (Figure 3).

Ces boules de vitamines sont généralement préparées en grande quantité, plus d'une quarantaine de boules à chaque fois, soit suffisamment pour remplir un sac de riz où elles seront stockées. Elles sont données aux éléphants en particulier lorsque les animaux participent à des tâches lourdes comme les travaux de débardage de bois. Ces opérations se déroulent sur plusieurs jours, voire même plusieurs semaines; il s'agit là d'un complément alimentaire indispensable que l'on emmène dans la forêt avec tous les autres équipements.

24 A contrario, lorsque l'animal apparaît trop fort, c'est le cas notamment des mâles en période musth (gnoi nya) on essaie de les affaiblir en leur donnant notamment du mak phak (Benincasa hispida). 
Figure 3 : Composition et fabrication des ya bam loung
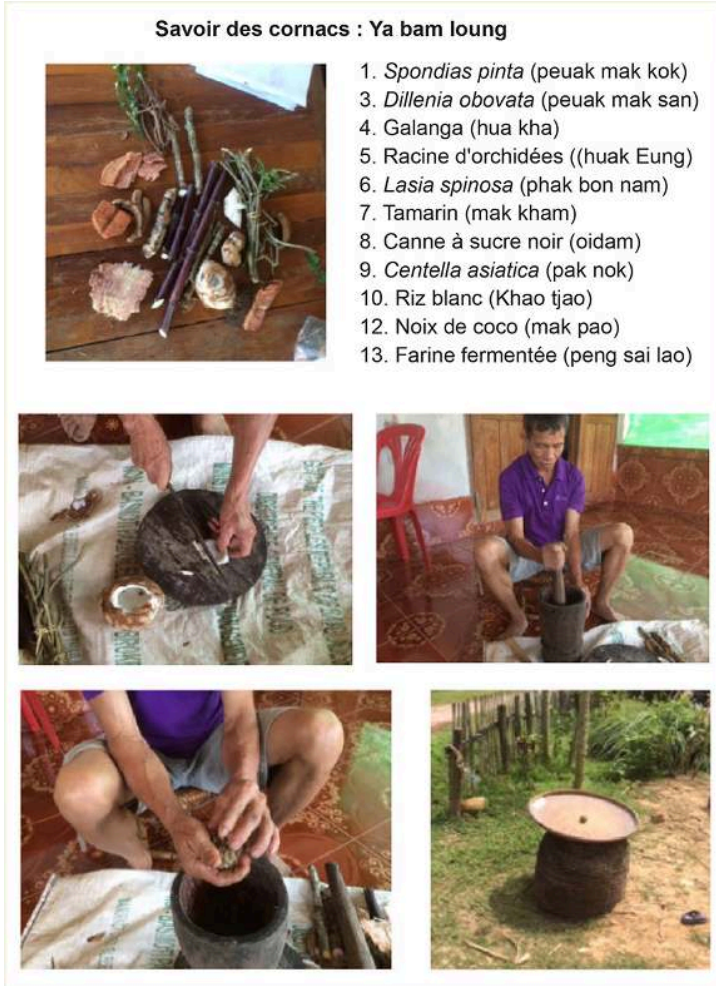

(c) N. Lainé 2016

Soulignons qu'en dehors des spécialistes chaque cornac ou propriétaire d'animaux interrogé dispose de sa propre pharmacopée, non écrite, qui est utilisée pour les soins quotidiens. Cornacs et propriétaires d'éléphants sont en effet particulièrement attentifs à la santé de leur animal, et opèrent quotidiennement une surveillance sur l'état physique des animaux ${ }^{11}$. Cette surveillance se déroule généralement dès le matin, en allant récupérer l'animal laissé libre la nuit dans la forêt. Il s'agit en premier lieu d'observer les différentes bouses autour des pachydermes (leur nombre, leur forme et couleur) pour avoir une idée de l'état de santé, de son humeur et estimer notamment si l'animal est prêt à aller travailler. Durant l'enquête, Lung Si me confiait que la première chose qu'il regardait en allant trouver son éléphant le matin sont les bouses, appelées koun khi xang. Chaque jour, s'il trouve cinq khi xang ou plus, c'est un signe de bonne santé, cela signifie que l'éléphant est assez fort et apte au travail. Au contraire, s'il en trouve moins de cinq, il saura que son éléphant a des problèmes, et pourra, le cas échéant, le laisser se reposer dans la forêt. En plus d'être attentifs au nombre de bouses produites quotidiennement, les cornacs prêtent aussi beaucoup d'attention à la forme et à la couleur de chaque khi xang. Plus le khi xang est foncé, plus l'animal sera mauvais voire agressif, alors que des bouses de couleurs claires (jaune) est un signe de bonne santé. Les pattes des éléphants sont aussi l'objet d'une attention particulière de la part des cornacs et propriétaires. Elles sont quotidiennement inspectées. Si un éléphant a de l'eau qui sort de ses ongles, il est considéré en bonne santé ; ce n'est pas le cas s'il a les pattes sèches.

Aussi pour soigner les blessures les plus courantes, la plupart des cornacs rencontrés disposent de leur propre préparation, en particulier pour soigner les abcès. C'est le cas de Tap dans le village de Ban Phonexay, âgé de 97 ans au moment de l'entretien ${ }^{12}$ qui 
m'a indiqué la concoction qu'il prépare lui-même. Il mélange des poils de poulets noirs (khoun khai dam), de la terre de termitière (din choum puak), et de l'écorce (puak) de thon kaiso (Schima wallichii). La pâte ainsi formée est appliquée directement sur l'abcès puis laissée sécher.

L'enquête de terrain consistait également à repérer si les hommes en charge des animaux leur fournissent des ingrédients que l'on trouve seulement au village, dont les éléphants n'ont pas accès dans la forêt. On trouve en effet des ingrédients propres aux villages, c'est-à-dire auxquels n'ont pas accès les éléphants sauvages, ou alors des aliments déjà transformés par l'homme: je pense au riz cuit par exemple, que l'on donne aux éléphants pour la première fois au moment de leur dressage, par un rite de séparation d'avec la mère ( $p a$ can xang). Sur ce point, il a été observé durant l'enquête que certains éléphants ne mangent pas de riz, la raison évoquée à chaque fois est que les parents de l'animal ne mangent pas de riz non plus $^{13}$. On trouve donc ici l'idée d'une transmission de pratiques ${ }^{14}$ alimentaires entre les éléphants. Sur ce point, il a été observé que lorsqu'une femelle met bas, elle sélectionne des plantes qu'elle place devant sa progéniture. En particulier de la canne à sucre (oidam) pour lui donner de l'énergie. Lors de la naissance d'un éléphant au village, plusieurs cornacs ont constaté que les éléphanteaux consommaient les excréments de leurs mères. Durant les premières semaines ou les premiers mois d'un jeune éléphant, plusieurs cornacs et propriétaires m'ont précisé qu'ils donnaient une plus importante quantité de nourriture à la mère (en particulier du riz cuit ou de la canne à sucre), car ils savent qu'une partie sera consommée par l'éléphanteau via les excréments de celle-ci). Plusieurs cornacs m'ont ainsi affirmé observer les éléphanteaux consommer les excréments de leur mère jusqu'à l'âge de trois ans. D'autres ont observé que les mères placent volontairement différentes lianes, feuilles ou fruits devant les jeunes animaux pour les nourrir, mais aussi, selon les cornacs, afin qu'ils en apprennent les différentes propriétés.

Puisant dans leur environnement immédiat, et tenant compte de l'état de santé des animaux, les communautés locales ont développé des formes uniques de connaissances médicales. Pour autant, si les informations présentés jusqu'alors font état de connaissances et d'un système de soin complexe concernant les principaux soins quotidiens des éléphants de village, il n'en demeure pas moins que l'ensemble des personnes en charge des éléphants rencontrés (mo xang, cornacs ou propriétaires) ont chacun insisté pour témoigner du fait que les éléphants ont une riche connaissance du monde de la forêt. Cette connaissance, m'ont-ils dit, s'expriment en recherchant des spécimens de plantes bien précis aussi bien pour se nourrir que pour se soigner. En effet, en plus de leurs connaissances locales, les cornacs et propriétaires d'éléphants insistaient tout autant sur la capacité des éléphants à se soigner par eux-mêmes. La forêt, m'ont-ils dit à plusieurs reprises, est l'équivalent de la pharmacie (hank ka ya) pour les éléphants, ils y trouvent nombre de médicaments. Lorsqu'ils sont malades beaucoup m'ont confié que les éléphants préfèreraient restaient seuls, sans voir d'humains que ce soit leur propriétaire ou le vétérinaire (satavapet), et que finalement la forêt était le lieu où l'animal était sabai (" en bonne santé »). Par exemple, dans leur quotidien avec ces animaux, il arrive de constater un éléphant être fatigué ou amaigri, surtout après plusieurs jours de travail en forêt. Leur morphologie peut aussi varier. Et tous sont d'accord pour dire qu'une fois la tâche accomplie, lorsqu'ils laissent leurs 
éléphants au repos, libres de déambuler dans la forêt, il ne leur suffit que de quelques jours pour retrouver leur poids de forme.

\section{Savoir des éléphants ? Aperçu de l'alimentation des pachydermes}

Plus important mammifère terrestre, herbivore, quotidiennement le régime alimentaire des pachydermes est composé de près de $250 \mathrm{~kg}$ de nourriture auquel se rajoutent 25 litres d'eau (Sukumar 1993). Les éléphants d'Asie disposent d'un système digestif assez peu efficace puisqu'ils n'assimilent que $40 \%$ des éléments nutritifs qu'ils ingèrent. Ce point explique en partie, avec son poids, la masse considérable d'aliment que ces animaux absorbent chaque jour. Les éléphants passent ainsi une importante partie de la journée à manger ou chercher des plantes, et leur alimentation varie considérablement selon le milieu où ils se trouvent. Face à cette importante quantité de nourriture, on dispose encore très peu de données sur la nutrition et l'alimentation des éléphants d'Asie dans les pays de l'aire de répartition.

Toutefois, au cours des dernières années, le régime alimentaire des pachydermes a été l'objet de plusieurs études dans plusieurs pays d'Asie. C'est le cas au Népal (Koirala et al. 2016), au Sri Lanka (Karunaratne \& Ranawana 1998), et en Inde où une étude a été conduite sur plusieurs années (1999-2006) (Joshi \& Singh 2008). Toutefois aucune étude systématique n'a été menée à ce jour. En Asie du Sud-Est, d'autres études ont eu des visées plus spécifiques. C'est le cas au Myanmar où des recherches ont cherché à déterminer le rôle des éléphants d'Asie dans la dispersion de graines (Campos-Arceiz et al. 2008), tandis que d'autres ont cherché à améliorer le bien-être des éléphants captifs. Cette dernière recherche a mis en évidence des différences significatives entre le régime alimentaire des mâles et des femelles. D'après les observations, ces dernières consomment une plus grande variété d'essences, tandis que les mâles se nourrissent plus fréquemment que les éléphants femelles (Himmelsbach et al. 2006). Enfin, à notre connaissance, une recherche en Thaïlande (Romain et al. 2014) a été menée sur le sujet afin de mieux comprendre l'alimentation des pachydermes en vue de concevoir un plan d'alimentation face à la perte d'habitat et aux restrictions concernant les espaces de fourrage laissés aux animaux. 
Figure 4 : Sortie en forêt en compagnie d'un cornac et de son éléphant Province de Sayabouri

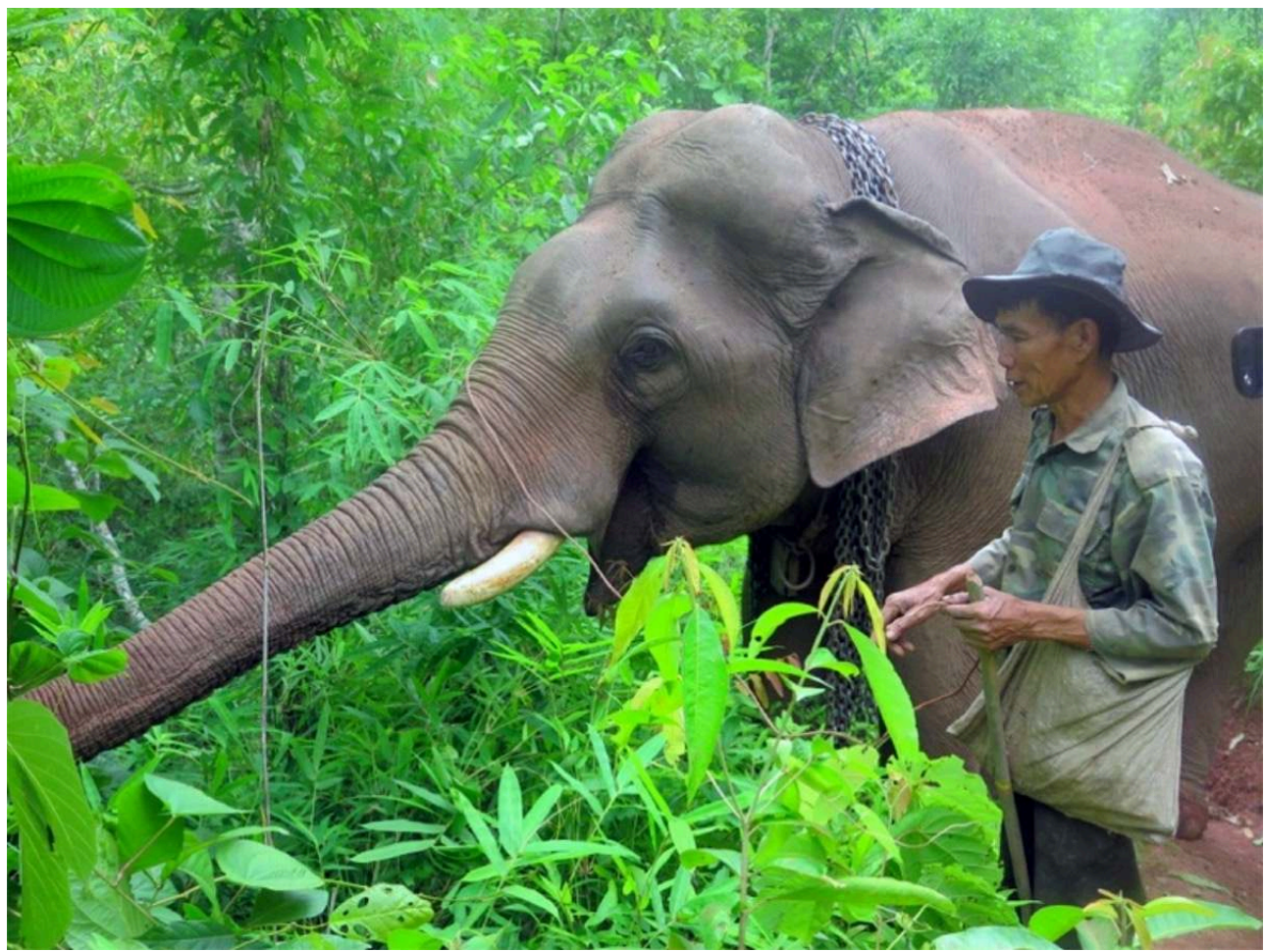

(C) N. Lainé 2016

31 L'enquête auprès des cornacs a d'abord permis de mettre en évidence des informations d'ordre général sur l'alimentation des éléphants. Il réside une grande variabilité, ce qui s'explique aisément par l'abondance et la disponibilité de certaines plantes ou fruits tout au long de l'année. Par exemple, durant la saison sèche (ladou leng), les éléphants consomment davantage de pousses de bambou (nor mai bon) et de bananes. Les interprétations fournies par les cornacs suggèrent qu'il s'agit là d'ingrédients qui contiennent une grande quantité d'eau. En fait, pour nombre de cornacs, en saison sèche les éléphants n'ont finalement pas vraiment le choix, et doivent se contenter de ce qui est disponible dans leur environnement immédiat. Ce n'est pas le cas durant la période de mousson (ladou phone) entre les mois de mai et septembre, où la végétation devient luxuriante et offre un plus large éventail de choix aux animaux. Les éléphants peuvent opérer une plus grande diversification de leur alimentation, même si pour une majorité de cornacs, leur préférence va toujours aux feuilles de bambou (mai bon). À noter que durant cette période de forte pluie, les éléphants consomment moins de bananes mais par contre ingurgitent les fleurs de bananiers (douak kwai).

Aux dires des cornacs et propriétaires d'éléphants, il existe également une importante variation du régime alimentaire selon le milieu et à la nature des différents travaux dans lesquels les animaux sont engagés. Par exemple, Lung Si m'a ainsi indiqué que lorsque son éléphant est au travail dans la forêt, il ne semble pas opérer de véritable choix quant aux essences consommées. Selon lui, son éléphant, un imposant mâle, a toujours très faim et doit manger en grande quantité15. A ce moment d'ailleurs, et seulement à ce moment, Lung Si donne du riz cuit à l'animal avec du sel (environ un kilogramme par jour) avec de la canne à sucre noire (oi dam). 

ingurgitées par leurs éléphants, plus d'un millier selon certains. Durant l'enquête, quelques-uns d'entre eux étaient cependant capables de distinguer les plantes prises dans le cadre de leur alimentation, nommée ahan xang, de celles indiquées comme faisant partie de leur régime médical, autrement dit les plantes qui soignent ya pua xang. Les cornacs qui ont accumulé au fil des générations des connaissances sur les habitudes alimentaires des éléphants ont ainsi pu «être attentif à l'ingestion de matériel inhabituel » comme l'avait souligné l'ethnobotaniste Hubert Gillet (1969) et comme le rappellent les vétérinaires Sabrina Krief et Hervé Hoste à propos de la façon de repérer des comportements d'automédication chez les animaux (2014). Parmi les essences qui sont revenues de manière récurrente, deux ont particulièrement attiré mon attention parce qu'elles ouvrent pour l'une à l'hypothèse d'une véritable pratique d'automédication, pour l'autre à celle d'un potentiel partage de savoirs avec les hommes. propriétaire de l'éléphant était assez clair sur les raisons de la prise par son animal. En effet, bien que disponible tout au long de l'année et en abondance, son éléphant n'en consomme qu'une seule fois l'an, toujours à la même période (au mois de janvier). L'animal n'en ingurgite qu'une dizaine de bouchées à chaque fois. Pour l'ensemble des cornacs interrogés, cette liane ne fait d'ailleurs pas partie du régime alimentaire régulier des éléphants. Aussi, la discussion engagée avec le propriétaire laisse émettre l'hypothèse d'un rôle de vermifuge par cette liane. D'ailleurs, comme il me l'a indiqué, depuis qu'il loue son éléphant dans un camp touristique, son animal est vermifugé par des injections. Et depuis lors, il n'a plus observé son éléphant consommer cette liane. Plusieurs études phytochimiques soulignent effectivement un rôle de vermifuge des feuilles de cette liane (Thyaga Raju et al. 2017, Vasudeva Rao \& Shanpru 1991).

Il en est de même concernant les racines (hak) de mak khunta (Harrisonia perforata, Rutaceae). Les racines de cette essence ont des vertus avérées contre la diarrhée (ya tai thong). Plusieurs études ethnobotaniques, et en particulier celle de Jules Vidal pour l'Indochine suggèrent des propriétés antimicrobiennes, antioxydantes, mais aussi antipaludiques et anti-inflammatoires. Cette racine est bien connue des cornacs et propriétaires rencontrés. A plusieurs reprises elle m'a été mentionnée comme faisant partie des essences données aux éléphants en cas de diarrhée, mais pas seulement. Les cornacs connaissent bien les feuilles de cet arbuste car elles sont bouillies et consommées en tisane en cas de diarrhée aigue chez les hommes. Il semble que nous ayons à faire là à un point de convergence d'usage de cette essence bien que, comme le soulignait Jules Vidal, si humains et animaux peuvent consommer les mêmes essences, les animaux consomment les matériaux bruts tandis que les humains les transforment.

Concernant les éléphants, dans le village de Ban Ha, Chanty Vanadee, ancien propriétaire d'éléphant aujourd'hui âgé de 82 ans rencontré dans le village de Ban Han m'a raconté une anecdote ${ }^{16}$. Il se souvient d'une après-midi en forêt avec son éléphant où il avait constaté que le ventre de ce dernier était particulièrement gonflé. En dépit de ces commandes, l'animal ne souhaitait rentrer directement au village, et ne l'écoutait pas. L'éléphant semblait rechercher quelque chose dans la forêt qu'il trouva lorsqu'il aperçut l'arbre thun khunta. A ce moment, Chanty dû même descendre du cou de l'animal qui a littéralement déraciné l'arbuste pour en consommer les racines. Rapidement après, Chanty se souvient que son animal faisait une importante quantité 
d'excréments, plus que d'habitude pour le remarquer. Dès lors, rentré au village, le ventre de l'animal n'était plus aussi gonflé et apparaissait en bonne santé.

\section{Conclusion}

L'enquête ethnographique réalisée auprès des cornacs, spécialistes (mo), et propriétaires d'animaux au Laos met au jour un système de soin complexe lié aux éléphants, qui inclut des éléments invisibles (médecine des rituels en lien avec les esprits) et visibles (médecine des remèdes par les plantes). Localement, cela engage les hommes en charge des animaux dans une surveillance quotidienne de leur état de santé et le cas échéant un ensemble de soins. Aujourd'hui pourtant ces savoirs sont peu ou pas mobilisés dans les actions de conservation de l'espèce. Au cours des dernières années, en s'appropriant les animaux (achat ou location), les parcs touristiques et centres de conservation ont largement transformé la gestion des pachydermes en mettant en avant la science vétérinaire moderne. S'appuyant sur une vision dualiste et 'éléphantocentrée' (Lainé 2018b) du bien-être et de la santé des animaux, cette gestion moderne laisse peu de place aux savoirs et connaissances locales. Elle gagnerait pourtant à inclure les connaissances et savoir-faire des populations qui vivent et travaillent quotidiennement avec ces animaux. Ces connaissances pourraient venir compléter les connaissances biomédicales, notamment pour le traitement des affections courantes telles les abcès. De manière générale, comme c'est le cas pour d'autres régions d'Asie, notamment en Inde du Sud (Lainé 2014, 2005) ou au Myanmar (Crawley et al. 2019), la lente disparition des éléphants dans les villages laotiens laisse présager une perte de savoirs des cornacs, qui constituent de véritables indicateurs sur l'état de biodiversité.

Par ailleurs, au niveau villageois, l'enquête souligne que les hommes en charge de ces animaux n'ont pas la prétention d'agir sur l'ensemble des aspects liés à la santé des pachydermes. Dans les villages Tai-Lue et Tai-Lao du Nord-Ouest du pays, la santé et les soins à prodiguer aux éléphants se basent sur des savoirs locaux -avec l'utilisation de plantes locales -, à quoi il faut ajouter un élément essentiel: le respect de la connaissance des éléphants eux-mêmes qui sont, selon leurs cornacs et propriétaires, capables d'automédication. C'est-à-dire que si les hommes leur fournissent les plantes nécessaires à une saine alimentation, ils sont conscients que les éléphants sont à même de les compléter en cas de besoin grâce à l'abondante biodiversité des espaces qu'ils traversent en leur compagnie. Ceci tient d'ailleurs à une des particularités de l'éléphant de village en Asie: sa circulation entre les différents espaces ${ }^{17}$ villageois (ban) et forestiers (pa). Historiquement, c'est cette circulation qui a permis le renouvellement $\mathrm{du}$ cheptel au niveau villageois ${ }^{18}$, tout en contribuant à la reproduction de l'espèce (Lainé 2018c). Elle permet également aux éléphants de jouer un rôle écologique fondamental (Lainé 2017b). Plus important encore, par cette circulation les pachydermes sont riches d'une somme de connaissances sur le monde de la forêt, auxquelles ils n'auraient plus accès s'ils restaient confinés au village ou dans un même espace (comme un camp touristique). Aussi, circuler entre le village et la forêt, et garder le contact avec des hardes sauvages leur permet de les conserver et de les transmettre au village. Cette transmission qui s'opère avec l'ensemble des éléphants, et en particulier avec ceux nés au village qui ne connaissent pas le monde de la forêt, pourrait dans une certaine mesure concerner les hommes. 

des éléphants (protection par le même esprit du foyer, cérémonie collective du baci) D'un autre côté, le recueil d'informations sur le régime alimentaire des éléphants a mis en évidence une possible convergence entre santé humaine et animale. C'est le cas concernant mak khunta. Cette convergence, qui mériterait d'être approfondie et étendue à d'autres essences, rappelle ce qu'écrivait Hubert Gillet en 1969 dans son cours d'ethnobotanique à propos de la cohabitation des hommes et des animaux et du comportement alimentaire des animaux sauvages : «il est possible que l'observation, faite par certains indigènes, du prélèvement occasionnel de certaines écorces d'arbres de savanes africaine, ait attiré leur attention sur ces arbres en tant que plantes médicamenteuses » (Gillet 1969: 19-20). Dans notre cas, si l'on tient compte de la longue cohabitation des hommes et éléphants en Asie depuis plusieurs millénaires ${ }^{19}$ et sur un même milieu, il est aisément possible d'émettre l'hypothèse que par leurs attachements réciproques, hommes et éléphants partageraient un ensemble de pratiques et de savoirs sur la base d'imitation ou d'emprunt interspécifique, comme cela a déjà été montré dans d'autres contextes et situations ${ }^{20}$. Et, supposer, comme ici avec les éléphants, que les groupes humains aient pu puiser dans l'observation et les connaissances des animaux invite nécessairement à repenser la manière de considérer les savoirs locaux. D'un point de vue épistémologique, ce renversement implique de ne plus accorder le primat des connaissances sur la biodiversité aux seuls humains mais, comme l'indiquait déjà Brunois (2005), à accorder une place prépondérante à l'interaction et à ses dynamiques pensées réciproquement.

\section{BIBLIOGRAPHIE}

Brunois F. 2005 - Pour une approche interactive des savoirs locaux : l'ethno-éthologie. Journal de la Société des Océanistes (120-121) : 31-40.

Campos-Arceiz A., Lin T.Z., Htun W. et al. 2008 - Working with mahouts to explore the diet of work elephants in Myanmar (Burma). Ecological Research $23: 1057$.

Condominas Georges 1976 - Essai sur l'évolution des systèmes politiques thaïs. Ethnos 41 (1-4) : 7-67.

Couture A. 2016 - La zoopharmacognosie. Voie alternative vers la découverte de nouveaux principes actifs. Thèse pour le doctorat d'état en pharmacie, Université d'Angers.

Crawley J.A.H., Lahdenpera M., Seltmann M.W., Htut W., Aung H.H., Nyein K., et al. 2019 Investigating changes within the handling system of the largest semi-captive population of Asian elephants. PLoS One 14 (1) : e0209701. https://doi.org/10.1371/journal.pone.0209701

Emourgeon C. 2010 - Usage et représentations de l'éléphant en Thailande : l'animal, le patrimoine, le symbole. Thèse en Ethnologie, Université Paris Ouest Nanterre La Défense.

Gillet H. \& Pujol R. 1969 - Cours d'ethnozoologie (1969-1970), 3ème cycle. Muséum national d'Histoire naturelle. 
Himmelsbach W., González Tagle M.O., Füldner K, Hoefle H.H. \& Wan Htun 2006 - Food plants of captive elehants in the Okkan reserved forest, Myanmanr (Burma), southeast asia. Ecotropica 12 : 15-26.

Hubback T.B. 1941 - The Malay Elephant. Journal of the Bombay Natural History Society 42 : 483-509.

Hunn E. 2012 - Ethnozoology. In : Anderson E.N., Pearsall D., Hunn E. \& Turner N. (Ed.) Ethnobiology. John Wiley and Sons : 83- 96.

Joshi R. \& Singh R. 2008 - Feeding behaviour of wild Asian Elephants (Elephas maximus) in the Rajaji National Park. The Journal of American Science 4 (2) : 34-48.

Karunaratne P. \& Ranawana K.B. 1998 - A preliminary study of the food preference of domesticated elephants (Elephas maximus) in Sri Lanka, Ceylan. J. Sci. (Bio. Sci.) 25 : 58-64.

Koirala R.K., Raubenheimer D., Aryal A., Pathak M.L., Ji W. 2016 - Feeding preferences of the Asian elephant (Elephas maximus) in Nepal. BMC Ecology 16 (1) : 54. Published 2016 Nov 17. doi: 10.1186/s12898-016-0105-9

Krief S. \& Brunois-Pasina F. 2017 - Interspécifité du pharmakon dans le parc de Kibale (Ouganda) : savoirs partagés, échanges, imités ou empruntes entre humains et chimpanzés. Cahiers d'Anthropologie Sociale, "Guérir, Tuer" : 112-135.

Krief S. \& Hoste H. 2014 - Les animaux pratiquent-ils la médecine par les plantes ? In : Morand S., Moutou F. \& Richomme C. (Ed.) Faune sauvage, biodiversité et santé: Quels défis ? Versailles, Quae : 75-89.

Labatut F. \& Suter I. 2010 - The Veterinary Care of Domesticated Elephants in Laos by a Mobile Veterinary Unit. Gajah 32 : 21-26.

Lainé N. 2005 - Des Éléphants et des Hommes. Approche anthropologique des relations homme/éléphant en Inde. Étude de cas : Éléments d'Ethnozoologie de l'Éléphant Captif (Elephas maximus) au Kerala (Inde du Sud). Master II Recherche «Individu et Société », Université de Monptellier 3, 194 p.

Lainé N. 2014 - Vivre et travailler avec les éléphants. Une option durable pour la survie de l'espèce. Thèse de doctorat en Ethnologie, Université Paris Ouest Nanterre La défense, 365 p.

Lainé N. 2017a - Surveiller les animaux, conserver l'espèce. Enjeux et défis de la surveillance de la tuberculose chez les éléphants au Laos. Revue Anthropologie des Connaissances, (2017/1) : 23-44.

Lainé N. 2017b - Travail interespèces et conservation. Le cas des éléphants d'Asie. Écologie et Politique 54 : 45-64.

Lainé N. 2018a - Elephant tuberculosis as a reverse zoonosis. Postcolonial scenes of compassion, conservation and public health in Laos and France. Medecine Anthropology Theory 5 (3) : 157-176.

Lainé N. 2018b - Asian elephants conservation: too elephantocentric? Towards a biocultural approach of conservation. Asian Bioethics Review 10 (4) : 279-293.

Lainé N. 2018c - Why the Khamti did not domesticate their elephants? Building a hybrid sociality with tamed elephants. In : Stépanoff C. \& Vigne J.-D. (Ed.) Hybrid Communities. Biosocial Approaches to Domestication and Other Trans-Species Relationships. New York, Routledge : 221-235.

Lainé N. 2018d - Coopérer avec les éléphants dans le Nord-Est indien. Sociologie du travail [En ligne] URL : https://journals.openedition.org/sdt/1953. Consulté le 15/12/2018.

Lainé N. 2018e - Bo mee xang pa kor bo mee xang (sans éléphant de forêt, il n'y a plus d'éléphant). Péninsule 75 (2017/2) :73-99. 
Maurer G. 2018 - Conservation de l'éléphant d'Asie (Elephas maximus) par l'étude des interactions entre humains et populations sauvages et semi-captives d'éléphants : une approche intégrée des dimensions démographiques, génétiques, économiques et socioculturelles (Ph.D. Thesis). Universitẻ de Montpellier.

Pottier R. 1973 - Note sur les chamanes et médiums de quelques groupes Thai. Asie du Sud-Est et Monde Insulindien 4 (1) : 99-109.

Pottier R. 2007 - Yû dî mî hèng, être bien avoir de la force. Essai sur les pratiques thérapeutiques lao. Paris, EFEO.

Romain S., Angkawanish T., Bampenpol P., Pongsopawijit P., Sombatphuthorn P., Nomsiri R. \& Silva-Fletcher A. 2014 - Diet composition, food intake, apparent digestibility, and body condition score of the captive asian elephant (Elephas maximus): a pilot study in two collections in Thailand. Journal of Zoo and Wildlife Medicine 45 (1) : 1-14.

Souvannavong 0. 1956 - Quelques pratiques de médecine traditionnelle. France-Asie (118-119) : 976-978.

Sukumar R. 1993 - The Asian Elephant: Ecology and Management. Cambridge, Cambridge University Press, $255 \mathrm{p}$.

Trautmann T.R. 2015 - Elephants and Kings: an Environmental History. Chicago, The University of Chicago Press, 304 p.

Thyaga Raju, Divya B.J., Suman B. \& Venkataswamy M. 2017 - The Traditional uses and Pharmacological Activities of Mucuna Pruriens (L) Dc: A Comprehensive Review. Indo American Journal of Pharmaceutical Research : 7 (01).

Vasudeva Rao MK \& Shanpru R. 1991 - Some plants in the life of the Garos of Meghalaya. In : Jain SK (Ed.) Contribution to Ethnobotany of India. Jodhpur, India, Scientific Publishers : 183-190.

Vidal Jules 1958 - La thérapeutique par les plantes au Laos. JATBA 5 (10) : 601-616.

Vidal Jules 1959 - Noms vernaculaires de plantes (lao, mèo, kha) en usage au Laos. Bulletin de l'École française d'Extrême-Orient 49 (2) : 35-608.

Zago M. 1972 - Rites et cérémonies en milieu bouddhiste lao. Roma, Università gregoriana.

\section{NOTES}

1. Sauf mention contraire, les données ethnographiques présentées dans cet article ont été collectées entre les mois juin et août 2016, à l'occasion d'un contrat de recherche postdoctorale FMSH/EFEO intitulé " La domestication de l'éléphant au Laos: recueil, analyse et valorisation d'un patrimoine menacé ». Je remercie le Centre EFEO de Vientiane m'a accueilli durant cette période.

2. En Asie ou en Afrique, différentes observations, souvent considérées comme anecdotiques, ont été constatées sur le terrain par des écologues ou environnementalistes. Dans les années 1970, l'écologiste Holly Dublin a observé une matriarche de l'espèce africaine (Loxodonta africana) parcourir 27 kilomètres hors de la brousse pour aller ingurgiter une plante de la famille des Borraginacées. Trois jours plus tard, cette femelle parturiente mettait bas (Couture 2016). Les hypothèses qui en découlent ont supposé que cette matriarche connût une ou plusieurs substances actives chez cette plante, laquelle a induit le travail d'accouchement. D'autres observations ont été faites en Asie sur les pachydermes. En Malaisie, il a été observé que des éléphants consommaient une légumineuse (Entada schefferi) aux supposées propriétés antalgiques permettant aux animaux d'effectuer des longues marches sans se fatiguer (Hubback 1941). De même, en Afrique comme en Asie, il a été observé à maintes reprises des pachydermes creuser 
dans le sol pour ingurgiter différents minéraux dans de l'argile. Ces pratiques ont été interprétées comme une recherche de propriété détoxifiante parmi les différents composants des plantes qu'ils consomment (Couture 2016). Très récemment encore en Inde, une éléphante indienne a été filmée en train d'ingérer du charbon de bois comme un remède à ses maux digestifs. La vidéo est vite devenue virale car l'animal donnait l'impression de fumer https:// www.youtube.com/watch?v=Gf8-M7oKgeM\&feature=youtu.be consulté le 13/12/2019.

3. Entretien réalisé avec Lung Sieng dans le village de Ban Viengkeo le 01/06/2016.

4. Le Laos a interdit les opérations de capture d'éléphants en 1989.

5. Cette méthode consiste à creuser une fosse, puis à l'abriter par de la végétation afin que les éléphants soient pris à l'intérieur. Pour déterminer la localisation des fosses, les cornacs s'appuyaient sur une connaissance fine des zones de passage migratoire des éléphants sauvages, laquelle renvoie pour les animaux une connaissance du milieu et de ses propriétés. Afin de ne laisser aucune échappatoire aux éléphants, le khoum xang doit être creusé sur un col entre deux montagnes, de préférence dans une terre riche en minéraux, que connaissent, recherchent et apprécient les éléphants sauvages, et que l’on nomme pong en laotien (Lainé 2018e).

6. À l'époque où les opérations de captures se pratiquaient encore, il existait une distinction entre les mo xang pa, les spécialistes des éléphants de forêt réputés plus dangereux, que les mo xang ban, les spécialistes qui ne peuvent traiter que les éléphants de village. Aujourd'hui, cette distinction entre spécialistes n'existe plus.

7. Cette pratique est également décrite par Emourgeon (2010) en Thaïlande.

8. Entretien réalisé avec Lung Si dans le village de Ban Phonexay le 01/07/2016.

9. Au Laos, cette méthode de l'œuf est également employée pour diagnostiquer l'état de santé des hommes et savoir à quel spécialiste se référer (Souvannavong 1956).

10. Pour un détail plus complet de l'opération de dressage au Laos, voir Maurer 2018.

11. Cette surveillance répond d'ailleurs aux enjeux de conservation de l'espèce discutés à une échelle globale (Lainé 2018a).

12. Entretien réalisé le 23/09/2016.

13. Entretien réalisé avec Lung Sieng dans le village de Ban Viengkeo le 01/06/2016.

14. Cette idée de transmission de pratiques entre éléphants fait écho à d'autres, observées dans le contexte indien. Ce type de transmission entre pachydermes concerne aussi bien leur collaboration au travail, notamment pour la réalisation des travaux de débardage de bois (Lainé 2018d)

15. En Inde, dans les camps de débardage de bois, j'avais également remarqué que les éléphants passaient leur temps à mastiquer des branches pendant qu'ils tiraient des grumes. Les cornacs me soufflaient qu'il s'agissait là de chewing-gum, comme ils le faisaient eux-mêmes en travaillant (Lainé 2014).

16. Entretien réalisé dans le village de Ban Ha, le 20/08/2016.

17. Ces deux espaces illustrent d'ailleurs la manière dont les populations taïes se représentent le monde qui, selon elles, repose sur une interdépendance et l'interaction à différents niveaux Les populations Taies sont organisées selon le muang (voir notamment Condominas 1976)

18. En laissant un animal libre de vaguer dans la forêt, on espère qu'il trouvera à s'accoupler avec l'un de ses congénères sauvages, qui donnera naissance à un nouvel individu qui pourra être capturé. Et ainsi de suite, dans un mouvement perpétuel de régénération et de maintien de l'espèce (Lainé 2018c).

19. Selon l'historien Thomas R. Trautmann les premières domestications de l'éléphant d'Asie dans la vallée de l'Indus remontent à entre -1000 à -500 av. J.-C. (Trautmann 2015).

20. D'un point de vue théorique, la poursuite de tels travaux ouvre par ailleurs la voie à des réflexions sur la genèse interspécifique de savoirs médicaux comme l'ont montrées Florence Brunois-Pasina et Sabrina Krief à propos de la société Botoro et la communauté de chimpanzés partageant leur territoire en Ouganda (Krief \& Brunois-Pasina 2016). Dans cette région où les 
mesures de conservation de la nature privent les humains d'accès aux ressources, les animaux eux deviennent les témoins et les garants de la mémoire humaine.

\section{RÉSUMÉS}

Cet article souhaite faire état d'un ensemble de pratiques ethno-vétérinaires des cornacs et des spécialistes $(\mathrm{mo})$ de la santé des éléphants au Laos. Il s'appuie sur une enquête ethnographique conduite dans les villages Tai-Lue et Lao dans la province de Sayabouri au Nord-Ouest du pays. Le corpus présenté souligne des ressemblances dans le traitement d'autrui tant sur le plan de la médecine des rituels que sur celles des remèdes. L'enquête montre également qu'au village les hommes en charge de ces animaux n'ont pas la prétention d'agir sur l'ensemble de la santé des pachydermes. Selon eux, les éléphants disposent d'une riche connaissance du monde de la forêt, et sont à même de compléter seuls les soins prodigués. Cet aspect m'a invité à élargir l'enquête pour m'intéresser au régime alimentaire des éléphants, à partir des connaissances des cornacs sur celui-ci. La conclusion insiste sur la nécessité d'inclure les savoirs locaux dans la conservation des éléphants, en complément des connaissances biomédicales. S'appuyant sur la longue cohabitation des hommes et des éléphants en Asie, elle suggère également des hypothèses sur le partage et la co-construction de savoirs médicaux entre humains et éléphants, ainsi que les renversements épistomologiques que cela implique.

This article aims to report on a set of ethnoveterinary practices of mahouts and elephant health specialists (mo) in Laos. It is based on an ethnographic survey conducted in the villages of TaiLue and Tai-Lao in Sayabouri province in the northwest of the country. The corpus presented highlights similarities in the treatment of others both in terms of ritual medicine and remedies. The survey also shows that in villages the men in charge of these animals do not claim to have any influence on the overall health of the pachyderms. According to them, elephants have a rich knowledge of the forest world, and are able to complete their treatments on their own. This aspect invited me to broaden the survey to focus on the elephant diet, based on the mahouts' knowledge of it. The conclusion emphasizes the need to include local knowledge in elephant conservation, in addition to biomedical knowledge. Drawing on the long cohabitation of humans and elephants in Asia, it also suggests hypotheses on the sharing and co-constuction of medical knowledge between humans and elephants, as well as their epistemological implications for apprehending local knowledge in anthropological inquiry.

\section{INDEX}

Mots-clés : pratiques ethno-vétérinaires, savoirs (humain et non humain), pharmacopée, éléphant, Laos

Keywords : ethno-veterinary practices, knowledge (human and non-human), pharmacopeia, elephant, Laos 


\section{AUTEUR}

\section{NICOLAS LAINÉ}

Post-doctorant, UMR 208, IRD/MNHN, "Patrimoines Locaux Environnement et Globalisation" Chercheur affilié, Laboratoire d'Anthropologie Sociale (LAS), Collège de France-CNRS-EHESS

Associé à l'Institut de recherche sur l'Asie du Sud-Est contemporaine (IRASEC, Bangkok) 